\title{
Effect of Prophylactic Fluconazole on the Frequency of Fungal Infections, Amphotericin B Use, and Health Care Costs in Patients Undergoing Intensive Chemotherapy for Hematologic Neoplasias
}

\author{
Andreas Schaffner and Maya Schaffner
}

Laboratory of Clinical Mycology, Division of Infectious Diseases, Department of Medicine, University Hospital, Zurich, Switzerland

\begin{abstract}
Fungal infections are a major problem in patients with hematologic malignancy. Attempts to reduce their frequency with antifungal agents have not been successful. A double-blind, controlled, single-center trial was conducted with 96 consecutive patients undergoing 154 episodes of chemotherapy. Patients received $400 \mathrm{mg}$ of fluconazole or placebo until bone marrow recovery or initiation of intravenous amphotericin B infusions. End points were amphotericin B use, fungal infection, stable neutrophil count $>0.5 \times 10^{9} / \mathrm{L}$, toxicity precluding further fluconazole use, and death. By KaplanMeier estimation, the time to initiation of amphotericin $B$ therapy was shorter in 76 patients treated with placebo than in 75 treated with fluconazole $(P=.003)$. Also, fluconazole reduced the number of febrile days by $20 \%(P=.002)$ and prevented oropharyngeal candidiasis $(1 / 75 \mathrm{vs.} 9 / 76, P=.018)$. The frequency of deep mycoses (8/76 vs. 8/75) and outcome were unaffected. Fluconazole did not have a favorable effect on infection-related health care costs and was associated with prolonged severe neutropenia $(P=.01)$.
\end{abstract}

Systemic fungal infections are a major problem in patients with hematologic neoplasias undergoing active chemotherapy for remission induction. About $5 \%-20 \%$ of intensive chemotherapy episodes are complicated by invasive mycoses [1-4], and deep fungal infection is found in $10 \%-40 \%$ of leukemia patients at autopsy [5-7]. In allogeneic bone marrow transplant recipients, an even higher frequency of mycoses has been reported from some centers [8]. Therefore, in most institutions, amphotericin B is used empirically in all high-risk patients with suspected fungal infection or unexplained fever refractory to antibacterial therapy [9-13]. Filtration of environmental air probably reduces the number of aerogenous infections with molds, such as Aspergillus species; however, this approach is expensive and not universally practicable, and it does not affect the frequency of endogenous infections with yeasts colonizing mucocutaneous surfaces [13]. Therefore, it would be desirable to use prophylactic regimens with antifungal agents that affect fungal colonization with yeasts and prevent candidiasis and aspergillosis, the most common invasive mycoses.

To our knowledge, no controlled study has clearly shown that antifungal prophylaxis affects outcome of intensive chemo-

Received 17 January 1995; revised 26 April 1995.

Presented in part: Biannual International Immunocompromised Host Society Meeting, Davos, Switzerland, June 1994.

Informed consent was obtained from each patient. This research was done with the approval of the ethical committee of the Department of Medicine, University of Zürich Medical School, and complied with the guidelines for clinical studies at our institution.

Pfizer Switzerland provided the salary for a study nurse and the study drug. Reprints or correspondence: Professor Andreas Schaffner, Dept. of Medicine, University Hospital, CH-8091 Zurich, Switzerland.

The Journal of Infectious Diseases 1995;172:1035-41 (C) 1995 by The University of Chicago. All rights reserved. $0022-1899 / 95 / 7204-0018 \$ 01.00$ therapy for hematologic neoplasias, reduces the frequency of invasive fungal infections in patients undergoing remission induction of hematologic neoplasias, diminishes the empiric use of amphotericin B, or reduces health care costs $[2,4,14-$ 18]. However, prophylaxis favorably affects mycosis-related morbidity and mortality in allogeneic bone marrow transplant patients who have a high frequency $(16 \%)$ of systemic candidiasis [8].

The present study was designed to investigate the effects of fluconazole on the need for intravenous (iv) amphotericin $B$ and on the frequency of fungal infections in a center in which aspergillosis is the predominant invasive mycosis [19]. In addition, the effect of prophylaxis on selected infection-related health care costs was studied. Secondary analysis end points included the duration of neutropenia and frequency of bacteremia in the study groups.

\section{Patients and Methods}

Patients. From 1 March 1990 to 30 August 1993, we screened all patients with acute myelogenous leukemia, lymphoblastic leukemia, or recurrent high-grade malignant lymphoma who were referred to our center for intensive chemotherapy that resulted in an expected granulocytopenia of $<0.5 \times 10^{9} / \mathrm{L}$ for $\geqslant 5$ days. All patients were enrolled and randomized during the study period, and patients were randomized again for each subsequent hospitalization for chemotherapy that resulted in a new episode of neutropenia. Patients were excluded if they had been treated with systemic antifungals within the preceding 7 days, had moderate or severe liver dysfunction, as shown by an elevation of aspartate amino transferase (AST) or alanine amino transferase (ALT) 3 times the normal value or a serum bilirubin level $>3 \mathrm{mg} / \mathrm{dL}$, or would not give informed consent. No patients were excluded due to other exclusion criteria (pregnancy, lactation, or age $<17$ years). 
Study protocol. Block randomization guaranteed that the placebo and study drug were evenly distributed over the study period. Eligible patients were randomly assigned in blocks of 10 to receive $400 \mathrm{mg}$ of fluconazole daily in capsules or as an infusion in 200 $\mathrm{mL}$ of saline or to receive an identically looking placebo, which was prepared by Pfizer (Orsay, France) and distributed by the hospital pharmacy. Stratified randomization guaranteed an equal distribution of underlying diseases and chemotherapy: Patients receiving chemotherapy without bone marrow transplantation for acute myelogenous leukemia and those receiving therapy for acute lymphoblastic leukcmia or salvage or dose-intensified chemotherapy followed by autologous bone marrow transplantation for highgrade malignant non-Hodgkin's lymphoma were stratified into 2 groups.

The study drug was administered at the patient's discretion either orally or by iv infusion. For patients with an estimated creatinine clearance $<40 \mathrm{~mL} / \mathrm{min}$, the dosing interval of the study drug was doubled; for those with clearance $<20 \mathrm{~mL} / \mathrm{min}$, it was tripled.

Prophylaxis was continued during hospitalization until one of the following end points was reached: stable neutrophil count $>0.5$ $\times 10^{9} / \mathrm{L}$, systemic antifungal therapy begun because of suspected or proven fungal infection, empiric iv amphotericin B therapy begun, toxicity precluding further administration of the study drug, or death. Some patients were withdrawn from the study because they were released from the hospital without signs of infection and with either rapidly increasing neutrophil counts or refractory leukemia and $<0.5 \times 10^{9}$ neutrophils $/ L$.

Three patients in the fluconazole group were replaced because of neutrophil counts $>0.5 \times 10^{9}$ cells $/ \mathrm{L}$ or for major protocol violations. The first patient was taken off the study drug for safety reasons (liver function test results $>3$ times normal value) before his neutrophil count declined to $<1.0 \times 10^{9}$ cells $/ \mathrm{L}$. The second patient inadvertently received commercial fluconazole, and the third patient was placed on iv amphotericin $B$ at the beginning of his first febrile episode. These patients were replaced during the study before the code was broken and, therefore, were not included in the efficacy analyses, with the exception of a complementary intention-to-treat analysis.

Patients were evaluated daily by 1 study nurse from the start of the study until hospital discharge. At baseline and weekly thereafter, the nurse inspected the oral mucosa, recorded lesions, and collected specimens for fungal cultures and microscopic examination. She also recorded daily compliance with administration of the study drug. Chest radiographs were obtained at baseline, and levels of creatinine, electrolytes, AST, ALT, and alkaline phosphatase were measured weekly during the study. Complete blood cell counts, including a differential white blood cell count, were obtained daily from the beginning of the study until hospital discharge. Body temperature was recorded twice daily. With each febrile episode (temperature $>38^{\circ} \mathrm{C}$ ), patients were given a physical examination, blood and urine were cultured, and chest radiographs were obtained

Antibacterial therapy. Antibacterial prophylaxis was not used; however, ceftazidime or piperacillin ( $\beta$-lactam antibiotics) combined with netilmicin or amikacin (aminoglycoside antibiotics) were initiated on an empiric basis according to recent guidelines $[11,12]$. Antibiotics were given to all patients who developed fever $>38.4^{\circ} \mathrm{C}$ (single measurement) or $>38^{\circ} \mathrm{C}$ (on 2 consecutive measurements) unless a febrile reaction to blood products was the suspected cause of fever. Empiric antibiotic therapy was adjusted according to microbiologic culture results and sensitivity tests, but patients always remained on a broad-spectrum antibacterial regimen consisting of two antibiotics. If fever of unknown cause persisted for $>48 \mathrm{~h}$, patients were given a second complete workup, including a physical examination, chest radiographs, and blood cultures. Clinical deterioration often resulted in changes to the empiric antibiotic regimen, at the discretion of the staff.

Antifungal therapy. Besides the study medication, patients received no routine antifungal prophylaxis. They were cared for in open wards without high-efficiency particulate air filters. Mouth washes with $0.2 \%$ chlorhexidine were done 6 times daily. Use of topical amphotericin B was permitted if staff suspected mucosal fungal infection, and it was used 5 times in the fluconazole group and 10 times in the placebo group. No other antifungal therapy was allowed. Empiric therapy with parenteral amphotericin B was initiated according to strict guidelines used for years at our center [19]: If a febrile episode persisted $\geqslant 96 \mathrm{~h}$ without response to empiric antibiotic therapy and had no documented etiology, patients underwent a reevaluation, including a clinical examination, blood cultures for fungi and bacteria, chest radiographs, and if the latter were not conclusive, a computed tomography (CT) scan of the lungs. Patients with chest radiographs or CT scans showing pulmonary infiltrates at the outset of a new febrile episode or with a CT scan confirming sinusitis were placed on amphotericin $B$ immediately. The drug was withheld for $72 \mathrm{~h}$ in patients for whom a CT scan of the chest excluded pulmonary consolidations or a pleural pathology.

The usual dosage of amphotericin B was $1 \mathrm{mg} / \mathrm{kg} /$ day accompanied for the first 3 days by $100 \mathrm{mg} / \mathrm{kg} /$ day of iv flucytosine given in three divided doses. At the beginning of iv amphotericin $\mathrm{B}$, the study drug was stopped to avoid possible antagonism $[13,20]$.

Evaluation of efficacy of antifungal prophylaxis. Primary end points were oropharyngeal candidiasis, deep fungal infection, and empiric use of iv amphotericin B. Oropharyngeal candidiasis was diagnosed with or without symptoms by the demonstration of yeast by microscopy or culture and by the presence of friable white oral lesions or erosions covered with exudative material. Systemic prophylaxis was considered to have failed if iv empiric amphotericin B was started or the diagnosis of definite or probable fungal infection was made. The following definitions were used: definite fungal infection-demonstration of hyphae or yeast in biopsy specimens or autopsy tissues or of yeast in blood cultures, probable fungal infection-demonstration of hyphae or yeast by microscopy as well as culture in material from lesions in not necessarily sterile sites, fever of undetermined origin not responding to $>96$ $\mathrm{h}$ of antibiotic therapy with pulmonary infiltrates showing on chest radiograph or CT scan, and fever of undetermined origin without pulmonary infiltrates not responding to $>96-\mathrm{h}$ of antibiotic treatment.

Analysis of infection-related health care costs. Cost factors related to the management of fever and infection complications were selected before the code was broken and quantified for the 151 evaluable chemotherapy episodes. These factors included the duration of hospitalization, the number of microbiologic and radiologic studies ordered, and the number and duration of systemic antifungal and antibacterial therapies prescribed. Prices were obtained from the hospital administration or pharmacy at the end of 
Table 1. Characteristics of study population at randomization.

\begin{tabular}{lccc}
\hline & $\begin{array}{c}\text { Placebo } \\
(n=76)\end{array}$ & $\begin{array}{c}\text { Fluconazole } \\
(n=75)\end{array}$ & $P$ \\
\hline Acute myelogenous leukemia & $54(71)$ & $55(73)$ & $.85^{*}$ \\
$\begin{array}{l}\text { High-grade malignant NHL } \\
\text { NHL with autologous bone }\end{array}$ & $22(29)$ & $20(27)$ & $.85^{*}$ \\
$\quad 7(9)$ & $8(11)$ & $.79^{*}$ \\
$\quad$ marrow transplantation & & & \\
First course of chemotherapy & $24(32)$ & $26(34)$ & $.86^{*}$ \\
$\quad$ slucocorticoid use dusing & $19(25)$ & $27(36)$ & $.17^{*}$ \\
Age, years \pm SD (range) & $40 \pm 13$ & $39 \pm 13$ & $.45^{+}$ \\
Neutrophil count $\left(\times 10^{\circ} / \mathrm{L}\right)$ & $(17-71)$ & $(17-67)$ & $.23^{+}$ \\
Creatinine level $(\mu$ mol/L) & $84 \pm 16$ & $5.00 \pm 8$ & $.92^{\dagger}$ \\
\hline
\end{tabular}

NOTE. NHL = non-Hodgkin's lymphoma. Data are no. (\%) of subjects, mean $\pm \mathrm{SD}$, or as otherwise noted.

* Fisher's exact test.

${ }^{+}$Mann-Whitney $U$ test.

August 1993. The costs in Swiss franks were converted to US dollars by applying an exchange rate of 1.0:1.15.

The results of the health cost analysis prompted a post-hoc analysis, after breaking the code, of the duration of neutropenia and frequency of bacteremia in the study population.

Statistical analysis. Before breaking the code, critical decisions (i.e., diagnosis of mycoses or declaring an episode nonevaluable) were discussed with an independent external infectious disease specialist not involved in the study. Results are given for all episodes except the three nonevaluable ones, which were included only in the intention-to-treat analysis. Kaplan-Meier analysis, $t$ tests (normal distributions), and Mann-Whitney $U$ tests (other than normal distributions) were done using the SATISTICA (StatSoft, Tulsa, OK) computer program. Frequency tables were computed by Instat (GraphPad, San Diego), Fisher's exact test (for numbers $<200$ ), and the $\chi^{2}$ test (for higher numbers). Results of two-tailed tests are given.

\section{Results}

Baseline characteristics. Among 173 consecutive eligible patient episodes, 167 were screened (6 courses of chemotherapy were missed for administrative reasons), and 154 patients were enrolled into the study ( 5 patients refused to participate, 4 had received systemic antifungal drugs in the preceding 7 days, and 4 had impaired liver function). Three of the 154 patient episodes were nonevaluable. Randomization of patient episodes resulted in an equal distribution of baseline characteristics. No significant differences were found between the 2 groups with regard to sex, weight, height (data not shown), age, primary diagnosis (acute myelogenous leukemia or lymphoma/ acute lymphatic leukemia with or without autologous bone marrow transplantation), use of glucocorticoids, kidney function, or baseline neutrophil count. The number of patients undergoing a first course of chemotherapy (table 1) and the pro- portion of patients with oral fungal colonization at study entry (see below) were comparable in both groups.

Effect of prophylaxis on amphotericin Buse. In 61 of 151 episodes, iv amphotericin was given according to preset guidelines: 25 of 75 patients in the fluconazole group and 36 of 76 in the placebo group received iv amphotericin B $(P=.098)$. While the proportion of patients receiving iv amphotericin B was not significantly different in the 2 groups, a Kaplan-Meier analysis of the time to failure (i.e., the time until the beginning of iv amphotericin B) showed that iv amphotericin B was administered earlier in the placebo group than in the fluconazole group (figure $1 ; \log$ rank test, $P=.003$ ). Patients receiving fluconazole prophylaxis remained on the study drug for a mean of 26.6 days (median, 25.0) compared with 20.28 days (median, 20.0) for the placebo group.

The finding that fluconazole prophylaxis delayed the need for iv amphotericin B was not changed when the 3 nonevaluable treatments were included in an intention-to-treat analysis $(n=$ 154; log rank test, $P=.003$ ). Patients in the placebo group received amphotericin $B$ for a total of 728 days, compared with 547 days for patients in the fluconazole group. The difference between the average duration of iv amphotericin B therapy (7.3 and 9.6 days in the fluconazole and placebo groups, respectively) did not, however, reach statistical significance ( $P=$ .238, Mann-Whitney $U$ test).

Because fluconazole was associated with a delay in starting amphotericin $B$ and because the decision to start iv amphotericin $B$ was made mainly on the basis of the extent and duration of fever (table 2), one would anticipate that patients in the fluconazole group had fewer febrile days during the study. Indeed, the percentage of febrile (fever $\geqslant 38^{\circ} \mathrm{C}$ ) days was significantly lower in patients receiving fluconazole than in the control group (figure 1). From this observation, it can be extrapolated that amphotericin B was given as planned.

Also in subgroup analyses, fluconazole prophylaxis delayed the use of iv amphotericin B in patients with acute myelogenous leukemia ( $n=109$; $\log$ rank test, $P=.004$ ), in patients who

Table 2. Indications for starting intravenous (iv) amphotericin B therapy in the 2 study groups.

\begin{tabular}{lccc}
\hline Indication & $\begin{array}{c}\text { Placebo } \\
(n=76)\end{array}$ & $\begin{array}{c}\text { Fluconazole } \\
(n=75)\end{array}$ & $\begin{array}{c}\text { Total } \\
(n=151)\end{array}$ \\
\hline FUO & 23 & $12^{*}$ & 35 \\
FUO plus infiltrate & 6 & 5 & 11 \\
Probable deep fungal infection & 1 & 2 & 3 \\
Deep fungal infection & 6 & 6 & 12 \\
$\quad$ Total & 36 & $25^{\dagger}$ & 61 \\
\hline
\end{tabular}

NOTE. Data are no. of subjects. Empiric amphotericin B was started in patients with fever of unknown origin (FUO) refractory to antibiotic therapy for $\geqslant 96 \mathrm{~h}$. In patients with $\mathrm{FUO}$ associated with new pulmonary infiltrate or clinical sinusitis confirmed by computed tomography scan, iv amphotericin B was started without delay at time of empirical antibiotic therapy.

* Fisher's exact test vs. placebo, $P=.05$.

${ }^{\dagger}$ Fisher's exact test, not significant. 
Table 3. Number of fungal infections per treatment group.

\begin{tabular}{lcc}
\hline & $\begin{array}{c}\text { Placebo } \\
(n=76)\end{array}$ & $\begin{array}{c}\text { Fluconazole } \\
(n=75)\end{array}$ \\
\hline $\begin{array}{l}\text { Mucocutaneous mycoses, oropharyngeal } \\
\quad \text { candidiasis }\end{array}$ & 9 & $1^{*}$ \\
$\begin{array}{l}\text { Deep fungal infections } \\
\text { Disseminated candidiasis } \\
\text { Aspergillosis }\end{array}$ & 4 & $0^{+}$ \\
$\begin{array}{l}\text { Zygomycosis } \\
\text { Probable aspergillosis } \\
\text { Total proven or probable deep } \\
\text { mycoses }\end{array}$ & 3 & 5 \\
$\quad \begin{array}{l}\text { Pulmonary infiltratcs with antibiotic- } \\
\text { refractory fever }\end{array}$ & 0 & 1 \\
$\quad \begin{array}{l}\text { Total definite, probable, and possible } \\
\text { deep mycoses }\end{array}$ & 8 & 2 \\
\hline
\end{tabular}

NOTE. Deep fungal infections included disseminated candidiasis due to Candida parapsillosis ( 1 case), septic shock with invasive candidiasis of stomach and the entire small intestine (1), chronic disseminated candidiasis (2), disseminated zygomycosis causing infarcts of liver and intestine (1), Aspergillus pneumonia with or without dissemination ( 8 definite and 3 probable).

$P=* .018$ or $^{\dagger} .12$, Fisher's exact test.

underwent a first course of chemotherapy ( $n=50$; log rank test, $P=.005)$, and in patients enrolled for the first time into the study $(n=96 ; P=.009)$.

Effect of prophylaxis on fungal infections. Patients receiving fluconazole prophylaxis developed significantly fewer mucosal infections with Candida species $(P=.018$; table 3$)$. Oral thrush, oral lesions, and yeast developed in 1 fluconazole patient and 9 placebo patients. In 2 patients, oropharyngeal candidiasis was present at randomization, and both were randomized to the fluconazole group. One of these patients healed within 1 week using the study drug alone; the other also received topical amphotericin B and healed within 2 weeks. The study design permitted topical use of amphotericin B for oral lesions. This had a limited effect on the interpretation of the prophylactic efficacy of fluconazole on oropharyngeal candidiasis. Among the 5 patients receiving amphotericin $B$ in the fluconazole group, 1 (mentioned above) had thrush at study entry and a second developed it during the study. In the 3 others, candidiasis was excluded as the cause of oral lesions by fungal cultures and direct microscopy. In contrast, 10 patients received topical amphotericin B in the control group, among whom 7 had microbiologically documented thrush.

The number of deep mycoses was not affected by fluconazole prophylaxis. There were no cases of invasive candidiasis during fluconazole prophylaxis, but there were 4 cases in the control group; however, there were 4 probable or definite fungal infections caused by molds in the placebo group and 8 cases occurred in the fluconazole group (two-tailed Fisher's exact test, $P=.36$; table 3 ). Two patients in each group died with a fungal infection. One patient in each group died with extensive pneumonia caused by Aspergillus fumigatus. An additional patient from the fluconazole group died of abdominal zygo- mycosis with extended intestinal and hepatic infarction. The second patient in the placebo group died with septic shock and negative blood cultures but with extensive invasive gastrointestinal candidiasis involving the stomach and the entire small intestine, as documented at autopsy. All remaining patients were cured of their fungal infections during hospitalization or after continuous antifungal therapy as outpatients (table 4). There was no significant difference between the 2 groups with respect to overall outcome.

Effects of prophylaxis on oropharyngeal colonization. The number of patients colonized by Candida species at study entry did not differ between the groups. During weekly microbiologic surveillance, the number of patients with oral Candida colonization increased in the placebo group but decreased in patients receiving fluconazole (table 5). About half of the patients had oral lesions or oral symptoms during the study, but frequently no association with candidiasis was found. The number of patients with an oral pathology was not significantly different between the 2 prophylaxis groups (table 5). De novo oral colonization with non-albicans species of Candida resistant to fluconazole was not observed during the study (table 5).

Clinical side effects and abnormal laboratory values. Adverse events are common in patients undergoing intensive chemotherapy for hematologic neoplasias. A cause-effect relationship between event and a particular drug is difficult to establish. Therefore, all registered adverse events for the fluconazole and placebo groups were compared, and no significant differences were found. There was no trend toward an increase in the number of skin reactions or pathologic liver function tests in patients receiving fluconazole (data not shown).

Effects of prophylaxis-related health care costs. Besides the costs for fluconazole and iv amphotericin B, possible effects of prophylaxis on predetermined health care costs related to

Table 4. Clinical outcome at end of hospitalization for patients given placebo or fluconazole.

\begin{tabular}{lcc}
\hline Outcome & $\begin{array}{c}\text { Placebo } \\
(n=76)\end{array}$ & $\begin{array}{c}\text { Fluconazole } \\
(n=75)\end{array}$ \\
\hline $\begin{array}{l}\text { Hematologic response to } \\
\text { chemotherapy, no deep fungal } \\
\text { infection }\end{array}$ & 62 & 60 \\
$\begin{array}{l}\text { Refractory leukemia, no fungal } \\
\text { infection }\end{array}$ & 2 & 2 \\
$\begin{array}{l}\text { Hematologic response, on systemic } \\
\text { antifungal therapy for suspected or }\end{array}$ & 7 & 10 \\
$\quad$ proven deep fungal infection* & 2 & 2 \\
$\begin{array}{l}\text { Died with deep fungal infection } \\
\text { Died without deep fungal infection }\end{array}$ & 3 & 2 \\
\hline
\end{tabular}

NOTE. Data are no. of subjects. For all comparisons $P>.1$ by Fisher's exact test.

* All infections were ultimately cured.

† In 3 patients, absence of fungal infection was confirmed at autopsy. In 1 control patient, no autopsy was done despite presence of sepsis syndrome of undetermined cause; clinically, no fungal infection was suspected. 
Table 5. Effects of fluconazole prophylaxis on oral colonization with Candida species and oral pathology.

\begin{tabular}{lccc}
\hline & $\begin{array}{c}\text { Placebo } \\
(n=76)\end{array}$ & $\begin{array}{c}\text { Fluconazole } \\
(n=75)\end{array}$ & $P^{*}$ \\
\hline $\begin{array}{l}\text { Before treatment } \\
\text { Direct microscopy positive for } \\
\quad \text { Candida species }\end{array}$ & 6 & 10 & .30 \\
$\quad \begin{array}{l}\text { Culture positive for Candida } \\
\text { species }\end{array}$ & 14 & 17 & .55 \\
$\quad$ Oral pathologic findings & & & \\
During treatment & 5 & 8 & .40 \\
$\quad$ Direct microscopy positive for & & & \\
$\quad$ Candida species & 14 & 1 & $<.001$ \\
$\quad$ Culture positive for Candida \\
$\quad$ species
\end{tabular}

NOTE. Data are no. of subjects. Samples for microbiologic studies were obtained by oral swab at study entry, weekly during study, and when therapy for oropharyngeal candidiasis was started.

* Fisher's exact test.

${ }^{\dagger}$ Most isolates were Candida albicans. Other Candida species were 1 each of $C$. lusitaniae, C. norvegensis, and C. tropicalis at study entry, 2 of $C$. glabrata during fluconazole prophylaxis, and 2 of C. krusei and 1 of C. glabrata during placebo treatment.

₹Epithelial defects (erosions or ulceration) or frank thrush (white fur) with or without symptoms

the management of fever and infection were evaluated with the expectation that a reduction in the number of febrile days or a reduced need for iv amphotericin B would reduce costs. No significant difference was found in the most important cost factor, duration of hospitalization, even though fluconazole patients were hospitalized an average of 1.6 days longer. Patients in the fluconazole group received iv amphotericin B on $23.9 \%$ fewer days but received parenteral antibiotics on $22.1 \%$ more days (table 6). This was unexpected in view of the reduced proportion of febrile days in patients treated with fluconazole (figure 1).
In accordance with the fewer febrile days in the fluconazole group, the number of examinations prompted by fever was also lower in that group (table 6). While none of the differences between the mean values of these parameters reached statistical significance, the fraction of hospital days on which iv amphotericin B or parenteral antibiotics were given was significantly different among the 2 groups $(P<.001$ for all four comparisons; table 6). In summary, we found that fluconazole did not reduce the limited number of evaluated infection-related health care costs, which tended to be higher $(12.3 \%, P=.060)$ in the fluconazole group (table 6).

Secondary analysis of the duration of neutropenia and the occurrence of bacteremia. The association of fluconazole administration with significantly more days of antibiotic therapy, despite fewer febrile days, suggested that fluconazole prophylaxis was associated with prolonged neutropenia. Antibiotics were not discontinued even if the patient was well and afebrile, if neutrophil counts were $<0.1 \times 10^{9} / \mathrm{L}[11,12]$. We therefore did a post-hoc secondary analysis. In the fluconazole group, the mean duration of neutropenia $\left(<0.5 \times 10^{9}\right.$ cells $\left./ \mathrm{L}\right)$ was 23.78 days $(95 \%$ confidence interval [CI], 20.75-26.81) compared with 19.5 days in the control group $(95 \% \mathrm{CI}, 17.29-$ 21.70; Mann-Whitney $U$ test, $P=.067)$. The difference between the duration of severe neutropenia $\left(<0.1 \times 10^{9}\right.$ cells $/$ L) was clear-cut, with no overlap of the $95 \%$ CIs between the 2 groups; it was 16.2 days $(95 \% \mathrm{CI}, 13.91-18.48)$ in fluconazole patients and 12.13 days $(95 \% \mathrm{Cl}, 10.61-13.65 ; n=151 ; P=$ $.01)$ in the placebo group.

The fluconazole group had a total of 2883 hospital days for 75 patient episodes. On 1784 of these days, neutrophil counts were $<0.5 \times 10^{9} / \mathrm{L}$, and on 1215 days they were $<0.1 \times 10^{9} \%$ L. In comparison, there were a total of 2796 hospital days for 76 patient treatment episodes observed in the placebo group; on 1482 of these days, neutrophil counts were $<0.5 \times 10^{9} / \mathrm{L}$, and on 922 days they were $<0.1 \times 10^{9} / \mathrm{L}\left(\chi^{2}\right.$ test, $P<.001$ for both comparisons). This finding may explain the higher

Table 6. Comparison of infection-related health care cost factors for study patients given placebo and patients given fluconazole.

\begin{tabular}{lcccc}
\hline & $\begin{array}{c}\text { Placebo } \\
(n=76)\end{array}$ & Cost $( \pm 95 \% \mathrm{CI})$ & $\begin{array}{c}\text { Fluconazole } \\
(n=75)\end{array}$ & Cost $( \pm 95 \% \mathrm{CI})$ \\
\hline Hostor & 36.8 & $25,912(22,863-28,961)$ & $38.4^{*}$ & $27,075^{*}(24,202-29,947)$ \\
$\begin{array}{l}\text { Days on fluconazole } \\
\text { Days on intravenous }\end{array}$ & 0 & 0 & 26.6 & $2157(1940-2375)$ \\
$\quad$ amphotericin B & 9.6 & $401(217-586)$ & $7.3^{*}$ & $305^{*}(154-457)$ \\
$\begin{array}{l}\text { Days on parenteral antibiotics } \\
\text { Blood cultures }\end{array}$ & 17.2 & $3726(3157-4294)$ & 21.0 & $4551^{\dagger}(3862-5241)$ \\
Chest radiographs & 7.9 & $414(342-487)$ & $7.3^{*}$ & $379^{*}(310-448)$ \\
Computed tomography scans & 4.61 & $476(396-557)$ & $4.01^{*}$ & $415^{*}(345-485)$ \\
$\quad 0.72$ & $629(304-955)$ & $0.64^{*}$ & $492^{*}(304-680)$ \\
$\quad$ Total for evaluated factors & & $31,559(27,754-35,364)$ & & $35,440^{*}(31,687-39,193)$ \\
\hline
\end{tabular}

NOTE. Data are mean costs in US dollars or mean no. of subjects, as appropriate, per treatment. Fees were those on 31 August 1993. $\mathrm{CI}=$ confidence interval.

${ }^{*}$ Mann-Whitney $U$ test, ${ }^{*} P>.1,{ }^{\dagger} P=.084 ;{ }^{\ddagger} P=.060$. 


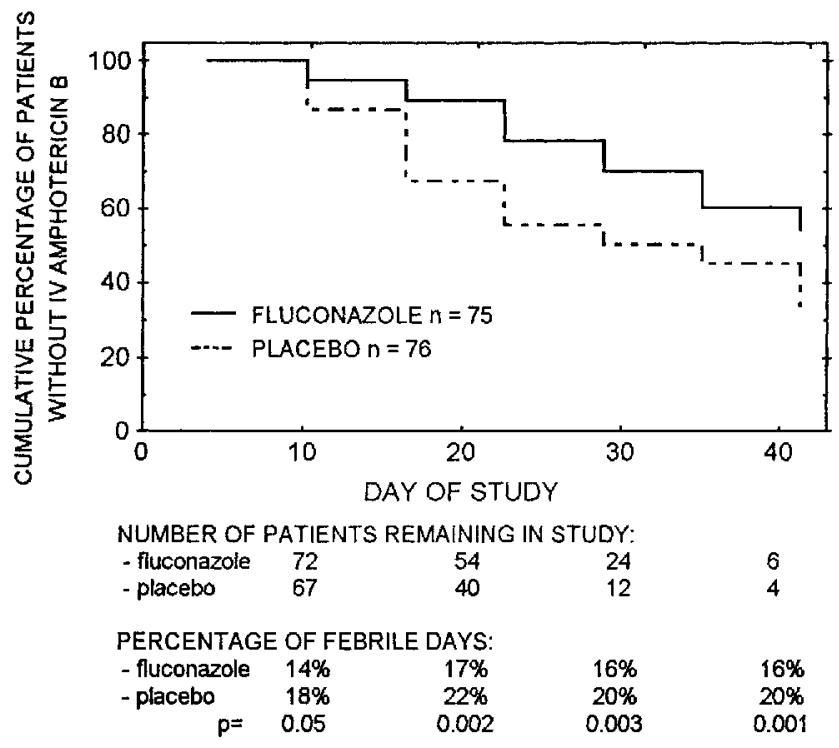

Figure 1. Kaplan-Meier plot showing \% of patients not receiving intravenous (iv) amphotericin $B$ at various times. Log rank test, $P=$ .003 . Shown in parallel are no. of patients remaining in study and cumulative \% of study days on which patients were febrile (temperature $\geqslant 38^{\circ} \mathrm{C}$ ).

proportion of days on parenteral antibiotics among the fluconazole group (1578/2883 hospital days) than among the placebo group (1307/2796 days) $(P<.001)$.

Because randomization was not stratified according to chemotherapy but only according to the type of hematologic neoplasia, it was important to assure that the association between prolonged neutropenia and fluconazole prophylaxis was not due to an inadvertent mismatch (e.g., in the type or intensity of chemotherapy). Therefore, extensive subgroup analyses were added. These analyses did not indicate that a mismatch with respect to intensity or cycle number of chemotherapy was responsible for the association between prolonged neutropenia and fluconazole. Patients with a blood neutrophil count $>0.1$ $\times 10^{9}$ neutrophils $/ \mathrm{L}$ after a single course of chemotherapy $(n$ $=147 ; P=.005)$, patients randomized for the first time into the study ( $n=96 ; P=.032$ ), or patients receiving a first course of chemotherapy ( $n=50 ; P=.004)$ had a significant association of prolonged severe neutropenia $\left(<0.1 \times 10^{9} / \mathrm{L}\right)$ and fluconazole prophylaxis. Also of note, the largest patient group receiving the same type of chemotherapy (a single course of daunorubicin combined with cytosine arabinoside) for acute leukemia had a strong and statistically significant association between prolonged neutropenia and fluconazole prophylaxis ( $n$ $=54 ; P=.025$ ).

Because severe neutropenia increases the susceptibility to bacterial infections, we also studied retrospectively whether fluconazole increased the number of bacteremic episodes in the study population. Among the 75 patients receiving fluconazole, 27 had bacteremia, and bacteremia occurred 16 times among the 76 patients receiving placebo $(P=.07)$. This difference was mainly due to an excess in the number of gramnegative bacteremia (11 vs. $4 ; P=.061)$ that were mainly caused by enteric gram-negative rods.

\section{Discussion}

In this single-center study, in which aspergillosis was the predominant mycosis in patients undergoing intensive chemotherapy for remission induction of hematologic neoplasias, the number of cases of systemic mycoses was not reduced by fluconazole prophylaxis. While no case of invasive candidiasis occurred in the fluconazole group, this advantage was offset by an increased number of definite or probable infections with molds. In agreement with results from comparable studies [2, $14,15]$, fluconazole prophylaxis in our study did not affect overall mortality or mortality from fungal infections and did not influence the immediate success of anticancer therapy. Also in agreement with results in other studies $[2,14,15,21]$, fluconazole prophylaxis reduced oral colonization with Candida species and the occurrence of oral thrush.

In contrast to the present study and others with a $<10 \%$ frequency of disseminated candidiasis [2, 15], a randomized, double-blind, multicenter study done in allogeneic bone marrow recipients found a significant reduction of deep mycoses during fluconazole prophylaxis and a reduced number of deaths with fungal infections [8]. The study had a high percentage $(16 \%)$ of systemic Candida albicans infections among placebo recipients, but mortality and overall prognosis were again not affected by fluconazole prophylaxis.

In our study, fluconazole reduced by $20 \%$ the number of febrile days and, thereby, delayed the need for iv amphotericin B. However, neither the proportion of patients requiring amphotericin B nor the mean duration of amphotericin B therapy was significantly reduced by fluconazole prophylaxis. This observation raises the question whether an important proportion of febrile episodes not responding to antibacterial agents during severe neutropenia is due to fungi. It is conceivable that the mucosal fungal flora of the gastrointestinal tract, after translocation facilitated by mucosal damage and neutropenia, is responsible for a systemic febrile inflammatory reaction in many of these patients, even without causing a clinically apparent mycosis. It is noteworthy in this regard that another placebocontrolled study recently suggested a reduction of fever by fluconazole prophylaxis in bone marrow transplant patients [22].

Since most studies have demonstrated that the efficacy of prophylactic antifungal agents is limited to infections with susceptible strains of Candida species and that such agents do not affect the frequency of systemic mycoses, mortality, or overall outcome, we questioned whether fluconazole prophylaxis would affect health care costs. Since fluconazole reduced the number of febrile days in our study, it would also be expected to reduce the number of antibiotic prescriptions and investigations 
aimed at the detection of the infectious causes of fever. However, fluconazole prophylaxis was unexpectedly associated with a trend toward increased use of parenteral antibiotics and did not decrease the number of investigations. It is a widely accepted policy to prescribe antibiotics for episodes of febrile neutropenia, despite defervescence, until granulocyte counts exceed $0.1 \times 10^{9}$ cells/L $[11,12]$. Prolonged neutropenia was therefore suspected as the reason that more antibiotics were prescribed despite the fewer febrile episodes in the fluconazole group.

Post-hoc analysis showed that the fluconazole patients had a $32 \%$ increase in the number of days with severe neutropenia $\left(<0.1 \times 10^{\circ} / \mathrm{L}\right)$. This secondary analysis also suggested a trend toward an excess of gram-negative bacteremia in the fluconazole group. It is not conceivable and none of the subgroup analyses indicated that the difference in the duration of severe neutropenia in the fluconazole and the placebo groups was due to a randomization mismatch. Nevertheless, future studies should prospectively address this question, especially because the mechanism responsible for the observed associations remains unclear. Others have made similar observations in a controlled study of ketoconazole prophylaxis in hematologic patients and speculated on the possibility of a drug interaction with antineoplastic drugs, resulting in an increased cytotoxic activity [4]. Another recent study demonstrated that fungal prophylaxis was associated with an increased risk for bacteremia without explaining its cause [23].

The routine prophylactic use of fluconazole in severely neutropenic patients reduces the number of infections with $\mathrm{Can}$ dida species, particularly oropharyngeal candidiasis, and delays the need for iv amphotericin B. However, this primary intervention does not affect the overall outcome for patients, presumably because fluconazole does not reduce the frequency of mold infections. Therefore, the routine use of fluconazole prophylaxis during intensive chemotherapy of hematologic neoplasias is not supported by the findings in this study. However, local epidemiologic data should be considered. Fluconazole prophylaxis should be considered in centers in which the frequency of deep infections caused by azole-susceptible yeasts is $>10 \%$. When mold infections are a problem, other strategies should be investigated, such as the use of compounds that are active against both molds and Candida species. Future clinical studies should prospectively investigate the effects of antifungal prophylaxis on the duration of neutropenia and the frequency of bacteremia.

\section{Acknowledgments}

We thank the medical and nursing staff of the Department of Medicine for their dedicated care of patients and contribution to the study.

\section{References}

1. Brown AE. Overview of fungal infections in cancer patients. Semin Oncol $1990 ; 17: 2-5$.
2. Winston DJ, Chandrasekar PH, Lazarus HM, et al. Fluconazole prophylaxis of fungal infections in patients with acute leukemia: results of a randomized placebo-controlled, double-blind, multicenter trial. Ann Intern Med 1993; 118:495-503.

3. Philpott-Howard JN, Wade JJ, Mufti GJ, Brammer KW, Ehninger G. Randomized comparison of oral fluconazole versus oral polyenes for the prevention of fungal infection in patients at risk of neutropenia. $J$ Antimicrob Chemother 1993;31:973-84.

4. Palmblad J, Lonnqvist $\mathrm{B}$, Carlsson $\mathrm{B}$, et al. Oral ketoconazole prophylaxis for Candida infections during induction therapy for acute leukaemia in adults: more bacteraemias. J Intern Med 1992;231:363-70.

5. Nosari A, Barberis M, Landonio G, et al. Infections in haematologic neoplasms: autopsy findings. Haematologica 1991; 76:135-40.

6. Pedraza MA. Mycotic infections at autopsy. A comparative study in two university hospitals. Am J Clin Pathol 1969;51:470-6.

7. Donhuijsen K, Samandari S. Tiefe Mykosen in Patienten mit Leulämien und Lymphomen. Dtsch Med Wochenschr 1985; 110;903-7.

8. Goodman $\pi$, Winston DJ, Greenfield RA, et al. A controlled trial of fluconazole to prevent fungal infections in patients undergoing bone marrow transplantation. N Engl J Med 1992;326:845-51.

9. Commers JR, Pizzo PA. Empiric antifungal therapy in the management of the febrile-granulocytopenic cancer patient. Pediatr Infect Dis $1983 ; 2: 56-60$.

10. EORTC International Antimicrobial Therapy Cooperative Group. Empiric antifungal therapy in febrile granulocytopenic patients. Am J Med 1989:86:668-72.

11. Pizzo PA. Management of fever in patients with cancer and treatmentinduced neutropenia. N Engl J Med 1993;328:1323-32.

12. Hughes WT, Armstrong D, Bodey GP, et al. Guidelines for the use of antimicrobial agents in neutropenic patients with unexplained fever. J Infect Dis 1990;161:381-96.

13. Schaffner A. Prophylaxis and treatment of fungal infections in cancer patients. Baillières Clin Infect Dis 1994;1:499-522.

14. White MH. Antifungal prophylaxis. Curr Opin Infect Dis 1994:6:737-43.

15. Menichetti F, DelFavero A, Martino $P$, et al. Preventing fungal infection in neutropenic patients with acute leukemia: fluconazole compared with oral amphotericin B. Ann Intern Med 1994; 120;913-8.

16. Wingard JR, Merz WG, Rinaldi MG, Johnson TR, Karp JE, Saral R. Increase in Candida krusei infection among patients with bone marrow transplantation and neutropenia treated prophylactically with fluconazole. N Engl J Med 1991;325:1274-7.

17. Meunier-Carpentier F, Cruciani M, Klastersky J. Oral prophylaxis with miconazole or ketoconazole of invasive fungal disease in neutropenic cancer patients. Eur J Cancer Clin Oncol 1983;19:43-8.

18. Denning DW, Donnelly JP, Hellreigel KP, Ito J, Martino P, van't Wout JW. Antifungal prophylaxis during neutropenia or allogeneic bone marrow transplantation: what is the state of the art? Chemotherapy 1992; 38(suppl 1):43-9.

19. Zimmermann Hosli MB, Stahel RA, Vogt P, Oelz O. Reduktion von invasiven Pilzinfektionen durch die empirische Anwendung von Amphotericin B in Patienten mit hämatologischen Neoplasien. Schweiz Med Wochenschr 1989;1 19:46-8.

20. Pahls S, Schaffner A. Aspergillus fumigatus pneumonia in neutropenic patients receiving fluconazole for infection due to Candida species: is amphotericin B combined with fluconazole the appropriate answer? Clin Infect Dis 1994; 18:484-6.

21. Ellis ME, Clink H, Enst $P$, et al. Controlled study of fluconazole in prevention of fungal infections in neutropenic patients with hematological malignancies and bone marrow recipients. Eur J Clin Microbiol Infect Dis 1994; 13:3-11.

22. Chandrasekar PH, Gaty CM. Effect of fluconazole prophylaxis on fever and use of amphotericin in neutropenic patients. Chemotherapy 1994:40:136-43.

23. Viscoli C, Bruzzi P, Castagnola E, et al. Factors associated with bacteraemia in febrile, granulocytopenic cancer patients. Eur J Cancer 1994; $30 \mathrm{~A}: 430-7$ 